\title{
The Detection of Oesophageal Varices Using a Novel, Disposable, Probe-based Transnasal Endoscope. A Prospective Diagnostic Pilot Study.
}

Sarmed S. Sami ${ }^{1}$, Krish Ragunath ${ }^{1}$, Emilie A. Wilkes ${ }^{1}$, Martin James ${ }^{1}$, Rodrigo MansillaVivar $^{1}$, Jacobo Ortiz-Fernández-Sordo'1, Jonathan White ${ }^{1}$, Amardeep Khanna ${ }^{1}$, Marina Coletta $^{2}$, Sunil Samuel ${ }^{1}$, Guruprasad P. Aithal ${ }^{1}$, Indra Neil Guha1.

${ }^{1}$ NIHR Nottingham Digestive Diseases Biomedical Research Unit, Nottingham University Hospitals NHS Trust and University of Nottingham, Nottingham, United Kingdom.

${ }^{2}$ Gastroenterology and Endoscopy Un V it, Fondazione IRCCS Ca' Granda Ospedale Maggiore Policlinico, Department of Pathophysiology and Transplantation, Università degli Studi di Milano, Milan, Italy.

\section{Correspondence:}

Dr. Indra Neil Guha, NIHR Nottingham Digestive Diseases Biomedical Research Unit, Nottingham University Hospitals NHS Trust and University of Nottingham, Level E, West Block, Queens Medical Center, Derby Road, NG7 2UH, Nottingham, UK.

Email: neil.guha@nottingham.ac.uk

Phone: +441159270609

Fax: +441159709955

Word count: 4,929

Number of figures: 5

Number of tables: 2

\section{Abbreviations:}


C-OGD Conventional oesophagogastroduodenoscopy

TNE Transnasal endoscopy

OV Oesophageal varices

VAS Visual analogue scale

AUROC Area under the receiver operating characteristic

$\mathrm{Cl} \quad$ Confidence interval

K Kappa

\section{Conflict of interest}

Professor Krish Ragunath is a research grant recipient from Intromedic Ltd, Seoul, South Korea. Other authors have no conflicts of interest to declare relevant to this publication.

\section{Financial support:}

Dr. S. S. Sami is funded by an Olympus-Core National Endoscopy Research Fellowship grant, Core Charity, United Kingdom (grant number: RB4803). This study was part funded by a grant from Intromedic Ltd, Seoul, South Korea.

\section{Trial registration:}

ISRCTN registry (identifier: 70595405). 


\section{Abstract}

Background and aims: Screening for oesophageal varices (OV) using conventional oesophagogastroduodenoscopy (C-OGD) is invasive and requires costly monitoring, recovery, and decontamination facilities. We aimed to evaluate the technical feasibility, acceptability and accuracy of a novel, portable, and disposable office-based transnasal endoscope (EG Scan ${ }^{\mathrm{TM}}$ ) compared to C-OGD as the reference standard.

Methods: This was a prospective cohort study. Consecutive adult patients with liver cirrhosis were invited to participate. All subjects underwent the two procedures on the same day performed by two endoscopists in a blinded design. Patients completed preference and validated tolerability (10-point visual analogue scale (VAS)) questionnaires on day 0 and day 14 post procedures.

Results: 45 out of 50 patients (90\%) completed both interventions. Mean age was 59 years and OV prevalence was $49 \%$.

Patients reported higher preference (percentage) and better experience (mean VAS) with EG Scan compared to C-OGD on day $0(76.5 \%$ vs. $23.5 \%, p<0.001 ; 7.8$ vs. $6.8, p=0.058$, respectively) and day 14 (77.8\% vs. $22.2 \%, p<0.001 ; 7.0$ vs. $5.5, p=0.0013$, respectively). Sensitivity and specificity of the EG Scan for the diagnosis of any size OV were 0.82 (95\%confidence interval (CI) $0.60-0.95)$, and 0.78 (95\% $\mathrm{Cl} \quad 0.56-0.93)$, respectively. Corresponding values for the diagnosis of clinically significant (medium/large) OV were 0.92 (95\% Cl 0.62-1.0), $0.97(95 \% \mathrm{Cl} 0.84-1.0)$, respectively. No serious adverse events occurred.

Conclusions: EG Scan accuracy was higher for the diagnosis of medium/large OV compared to any size OV. Patients' preference and overall experience of the EG Scan was favourable compared to C-OGD 14 days after procedures.

Abstract word count: 248. Keywords: Transnasal; endoscopy; screening; varices. 
Key points

- Conventional oesophagogastroduodenoscopy (C-OGD) is invasive and may not be cost-effective as a screening tool for oesophageal varices (OV).

- The EG Scan ${ }^{\mathrm{TM}}$ is a novel portable and disposable transnasal endoscope which may be used as a rapid office-based screening test.

- Novel data from this study demonstrate that the EG Scan ${ }^{\mathrm{TM}}$ was safe, successful, and appears to be acceptable to patients.

- EG Scan ${ }^{\mathrm{TM}}$ was accurate in the detection of clinically significant OV.

\section{Introduction}

Screening for oesophageal varices (OV) using conventional oesophagogastroduodenoscopy (C-OGD) followed by primary prophylaxis is currently recommended for all patients with cirrhosis $[1,2]$. However, the cost-effectiveness of C-OGD as a screening tool in this context is doubtful [3]. The prevalence of OV is highly dependent on disease severity; and ranges from as low as $40 \%$ in patients with Child-Pugh class $A$ to $70 \%$ in those with Child-Pugh class B/C [4]. This implies that a significant proportion of patients currently undergo serial negative endoscopies with no clinical benefit. Thus, having a more effective strategy for screening and stratifying which patients need C-OGD will have direct implications for patients and the healthcare system [1,2].

A large number of non-invasive tests for the detection of OV have been evaluated[5], but none have substituted C-OGD as the standard reference tests in current clinical practice[1,5]. C-OGD is performed in a hospital setting usually under conscious sedation to increase comfort and tolerability [6]. This leads to loss of work time on the day of procedures and the need for the patient to be accompanied home afterwards. Moreover, performing COGD has many other logistical and cost considerations as it requires patient monitoring, 
experienced nursing staff, recovery rooms and decontamination equipment [6]. Therefore, un-sedated ultrathin transnasal endoscopy (TNE) has been evaluated in this setting; however, the available devices still require decontamination facilities and are usually performed in dedicated endoscopy suites [7]. The need for controlled trials on office-based, portable TNE screening for OV was highlighted in recent guidelines from the British Society of Gastroenterology [2].

The second generation E.G. $\operatorname{scan}^{\mathrm{TM}}$ (EG Scan $\left.{ }^{\mathrm{TM}} \mathrm{II}\right)$ is a novel transnasal video endoscope which is portable, lightweight, sedation-free, and has a disposable probe which eliminates the need for decontamination equipment; hence, may overcome the limitations of C-OGD for use as an office-based rapid screening test. Aedo et al [8], evaluated the accuracy of an early prototype of this device (ESOScan ${ }^{\mathrm{TM}}$ ) in the detection of various upper gastrointestinal pathologies with promising results. However, this early model had no air insufflation capability and did not allow examination in the retroflexion position, which may limit its utility for the assessment of OV size and extent. Moreover, the impact of this technique on patients' preference and tolerability was not evaluated. The purpose of this study was to assess the technical feasibility, acceptability, and accuracy of the EG Scan ${ }^{\mathrm{TM}}$ II system for the detection of OV in patients with liver cirrhosis.

\section{Materials and methods}

\section{Study design and settings}

This was a prospective diagnostic cohort study in a tertiary referral centre (Nottingham University Hospitals NHS Trust, Nottingham, United Kingdom) and all procedures were performed between July $31^{\text {st }} 2012$ and $25^{\text {th }}$ February 2014. All the participants provided written informed consent and the study received approval from the East Midlands Nottingham 1 research ethics committee. The trial was prospectively registered on the ISRCTN registry (identifier: 70595405). This research was conducted and reported 
according to the standards for the reporting of diagnostic accuracy studies (STARD) statement [9].

\section{Participants}

Consecutive adult patients with known liver cirrhosis (confirmed by clinical, radiological, and/or histopathological criteria) who are scheduled to undergo C-OGD for screening or surveillance of OV at our hospital were invited to participate in this study. We excluded patients with recurrent epistaxis (more than once a week); complete nasal obstruction; disease of the nasal cavity; prior history of variceal bleeding (defined as haematemesis or melaena requiring hospital admission); and those who had band ligation therapy in the past 12 weeks.

\section{Interventions}

All patients underwent TNE first using the EG scan followed by C-OGD on the same day. All EG Scan procedures were performed by a single experienced endoscopist (performed over 1000 C-OGD procedures), but had limited prior experience in using the EG Scan device (performed 3 procedures on 3 volunteers). The operator was blinded to the results of any previous investigations. C-OGD procedures were performed by another two experienced endoscopists who were blinded to the findings of the EG Scan procedure. The size of OV was graded according to the American Association for the Study of Liver Diseases consensus classification system into either small or medium/large OV (small OV defined as small straight varices that usually disappear on air insufflation; medium OV defined as enlarged tortuous varices occupying less than one third of the lumen; and large OV defined as coil-shaped varices occupying more than one third of the lumen) [1]. Patients were asked to fill in validated questionnaires on day 0 and day 14 after the procedures.

\section{The EG Scan ${ }^{\mathrm{TM}}$ /I system}


The second generation EG Scan system (Intromedic Co. Ltd., Seoul, South Korea) (figure 1) consists of four main components:

1. A single use probe (diameter $3.4 \mathrm{~mm}$, length $1088 \mathrm{~mm}$ ) which is made from human compliance plastic and contains:

a) Capsule at the tip (diameter $6.1 \mathrm{~mm}$ ) which produces images at a speed of 30 frames per second with a $125^{\circ}$ field of view and $3-50 \mathrm{~mm}$ view depth. The camera capsule incorporates a complementary metal-oxide semiconductor (CMOS) image sensor with $400 \times 400$ pixel array.

b) Bending wire to facilitate tip deflection (bending angle $160^{\circ}$ up $/ 160^{\circ}$ down).

c) Data connector to transmit captured images from the capsule to the processor.

d) Air insufflation channel.

2. A hand-held controller with an up/down lever to facilitate tip deflection. The probe is attached to the controller.

3. An image processor which incorporates a free air displacement system (5L/min). Images are transmitted from the tip of the probe to the processor and then displayed on a laptop computer screen via USB connection.

4. An air tube which connects the processor to the probe in order to facilitate air insufflation.

\section{Endoscopy procedures}

The EG Scan procedure was performed in an outpatient clinic room with the patient sitting in a chair next to the physician's desk. Prior to commencing the procedure, participants were given a $100-\mathrm{ml}$ liquid oral drink which is a mixture of water; orange cordial flavouring; a mucolytic (10 mls of $200 \mathrm{mg} / \mathrm{ml} \mathrm{N}$-acetylcysteine, Parvolex®, Recipharm Ltd, Ashton under Lyne, UK); and an anti-foaming agent $(1 \mathrm{ml}$ of Simeticone $40 \mathrm{mg} / \mathrm{ml}$, Infacol $\AA$, Forest Laboratories UK Ltd, Dartford, UK) in order to improve the visualization of the mucosa. A topical aerosol spray (Lidocaine Hydrochloride 5\% and Phenylephrine Hydrochloride 0.5\%; Pharmacy Production Unit, Queens Hospital, Burton on Trent, UK) was applied to the 
patient's nares (3-4 sprays) 3-5 minutes prior to the procedure. The endoscope was introduced into the right or left nares and advanced into the proximal oesophagus under direct vision. The operator carefully inspected the oesophagus and the gastroesophageal junction both in forward and retro-flexion views.

Following the procedure, the probe was disconnected from the hand-held controller and discarded. The system was dismantled and stored in the accompanying suitcase (figure 1A). The C-OGD procedures were performed at our endoscopy suite using a 9.8-mm diameter high definition endoscope (GIF-H260, Olympus Keymed, Essex, UK). The procedure was performed after either a topical anaesthetic spray was applied to the posterior pharynx (5-10 sprays, Lidocaine 10mg/dose, Xylocaine, AstraZeneca, Luton, UK) or under sedation with intravenous midazolam (Hameln pharmaceuticals Itd, Gloucester, United Kingdom), according to patient preference as per standard practice in the UK.

\section{Patient questionnaires}

All questionnaires were administered by the study coordinator and completed by patients after the endoscopist left the examination room. Patients' tolerability was measured using a validated 10-point visual analogue scale (VAS) [10]. Patients were asked to describe their overall experience after each procedure by placing a cross on a line from "0" (worst ever experience) to "10" (best ever experience). We also measured the degree of gagging, choking, discomfort, and nasal pain (EG Scan only) using the same scale where "0" is none and "10" is severe. After completion of both procedures, patients were asked to choose which procedure they would prefer to have in the future if clinically indicated (EG Scan, or COGD, or either). Tolerability and preference questionnaires were completed on the same day (day 0 ) and 2 weeks (day 14) after procedures (using prepaid self-addressed return envelopes) to allow for the complete resolution of sedation effects and assess long term acceptability.

\section{Outcome measures}


Study outcome measures included:

1. The technical feasibility, quality and safety of the endoscopic examinations (EG Scan and C-OGD) measured as: (i) rate of successful intubation (the ability to traverse the upper oesophageal sphincter and visualize the oesophageal mucosa) classified as successful or unsuccessful; (ii) rate of complete evaluation (visualization of the whole oesophagus and identification of the landmarks: squamocolumnar junction, gastroesophageal junction [upper margin of gastric folds with stomach deflated] and the diaphragmatic hiatus) classified as complete $=$ all three landmarks identified, incomplete = some landmarks identified, or unsuccessful; (iii) duration of examination (time from intubation to extubation for the EG Scan; and (iv) rate of adverse events including: withdrawal of consent, bleeding, perforation, or need for hospitalization assessed immediately and 14 days (telephone call from the study coordinator) after the procedures in all subjects.

2. Patients' acceptability of both procedures measured using validated questionnaires as detailed above.

3. The real time accuracy of EG Scan (index test) in detecting any size OV and clinically significant (medium/large) OV using C-OGD as the reference standard. This was measured using sensitivity, specificity, and area under the receiver operating characteristic (AUROC) curve.

4. Inter-observer agreement on the EG Scan and C-OGD findings (presence and grade of OV) measured using the Kappa statistic. Anonymized video clips were recorded for both EG Scan $(n=45)$ and C-OGD $(n=45)$ procedures, then edited to a length of $1-2$ minutes. Nine blinded assessors ( 3 hepatologists, 3 gastroenterologists, and 3 trainee gastroenterologists) evaluated all the videos independently of each other and in random order. Prior to the evaluation stage, all observers received a 20 -minute training session during which they reviewed 5 representative video clips and 10 still images for the EG 
Scan (first 10 minutes), followed by the same number of videos and images for C-OGD (second 10 minutes) in order to demonstrate the presence, absence, and different grades of OV.

\section{Statistical analysis}

This was a pilot study to collect data which will help inform power calculations for future trials. We assessed a group 50 patients, based on a realistic recruitment target over 18 months. We used paired t-test (normally distributed differences) and Wilcoxon signed rank test (non-normally distributed differences) to compare measurements of continuous variables between EG Scan and C-OGD procedures. The Chi-squared or Fisher exact tests were used to compare categorical variables. A p value of less than 0.05 was considered statistically significant. The kappa $(\mathrm{K})$ statistic was calculated to measure the degree for agreement after correcting for chance agreement [11]. The interpretation of k-values was done according to the guidance provided by Landis and Koch where values $<0$ indicate no agreement; $0-0.20$ = slight; $0.21-0.40$ = fair $; 0.41-0.60=$ moderate; $0.61-0.80$ = substantial; and $0.81-1=$ almost perfect agreement [12]. Statistical computations were performed using the Stata version 12.0 software (Stata Corporation, College Station, Texas, USA).

\section{Results}

\section{Baseline Characteristics}

50 patients were recruited to the study and 45 (90\%) completed both procedures. 43 out of 45 patients (93\%) had compensated cirrhosis. Prevalence of OV was $49 \%(27 \%$ medium/large) (table 1).

\section{Technical feasibility, quality and acceptability}

3 patients failed EG Scan (6\%) and 2 failed C-OGD (4\%), p=0.882 (figure 2). 26 out of 45 (58\%) C-OGD procedures were performed under sedation using intravenous midazolam 
(mean dose $3.2 \mathrm{mg} ;+$ +-0.9) with or without intravenous pethidine (median dose $0 \mathrm{mg}$; IQR 0$50)$.

The mean duration of EG Scan examination was 8.1 minutes (+/-2.4). Results of technical feasibility, quality, safety and acceptability are presented in table 2. Data on procedure preference and experience (VAS) are also shown in figure 3 and figure 4, respectively. Four patients (8.5\%) experienced minor self-limiting epistaxis. No serious adverse events occurred.

\section{Accuracy and interobserver agreement}

Sensitivity, specificity and AUROC of the EG Scan for the diagnosis of any OV were 0.82 (95\%confidence interval (Cl) $0.60-0.95), 0.78(95 \% \mathrm{Cl} 0.56-0.93)$, and $0.80(95 \% \mathrm{Cl} 0.68-$ 0.92), respectively. Corresponding values for the diagnosis of medium/large OV were 0.92 (95\% Cl 0.62-1.0), $0.97(95 \% \mathrm{Cl} 0.84-1.0)$, and $0.94(95 \% \mathrm{Cl} 0.86-1.0)$, respectively.

For EG Scan, $\mathrm{K}$ values (all observers) for the presence of any OV, small OV and medium/large OV were $0.45(95 \% \mathrm{Cl} 0.40-0.49), 0.19(95 \% \mathrm{Cl} 015-0.24)$ and $0.47(95 \% \mathrm{Cl}$ 0.42-0.52), respectively. For C-OGD, corresponding values were $0.54(95 \% \mathrm{Cl} 0.49-0.59)$, $0.22(95 \% \mathrm{Cl} 0.17-0.26)$ and $0.67(95 \% \mathrm{Cl} 0.62-0.71)$, respectively. Example findings of $\mathrm{EG}$ Scan are shown in figure 5.

\section{Discussion}

\section{Principal findings}

This is the first study evaluating the feasibility and clinical utility of the EG Scan II device as an office-based screening tool for OV in a selected cohort of patients with liver cirrhosis. We demonstrate that the trans-nasal technique with EG scan can be performed safely and it is acceptable by patients compared to C-OGD. 
Unsedated TNE techniques have been developed in an attempt to address some of the limitations of C-OGD with regards to tolerability, acceptability, and the need for sedation with costly recovery and monitoring suites [13]. We recently reported results from a systematic review and meta-analysis of 34 studies (6,659 patients) demonstrating that there was no significant difference in technical success rate of currently available TNE devices $(<5.9 \mathrm{~mm}$ insertion diameter) compared to C-OGD [13]. Moreover, patients preferred TNE over C-OGD in all 10 studies (1,418 patients) where this outcome was measured [13]. Success rate of EG Scan was not significantly different from C-OGD in the current study (table 2) and preference data were also highly encouraging. Patients reported higher preference for EG Scan compared to C-OGD on both day $0(76.5 \%$ vs. $23.5 \%, \mathrm{p}<0.001)$ and day $14(77.8 \%$ vs. $22.2 \%, \mathrm{p}<0.001)$ after procedures independent of the use of sedation for C-OGD (table 2). The patients' experience (VAS) was better with EG Scan; however, there was no significant difference in experience when sedation was used for C-OGD (table 2). The use of propofol for C-OGD is likely to make it even more attractive to patients compared to EG Scan. The EG Scan performance in this study appears to be favourable compared to other currently available minimally invasive markers. Oesophageal capsule endoscopy is a well-tolerated and promising tool but reports on sensitivity (for the detection of any size OV) have been conflicting and ranged from $63 \%$ to $100 \%$ in different studies [14]. This may be related to the inability to insufflate air during examination with oesophageal capsule endoscopy. Moreover, the cost-effectiveness of this technique compared to C-OGD remains doubtful [15].

While the EG Scan was accurate in detecting clinically significant (medium/large) OV, it was inferior to C-OGD when small OV were included in the analysis. This finding could be due to a limitation of the EG Scan imaging quality or could equally be a result of poor agreement between the EG Scan and C-OGD operators on the presence or absence of small OV. All operators received training and followed a standardized protocol for examination and image documentation in order to minimize observer bias. However, it is well known that OV size and shape can vary significantly depending on the amount of air insufflation and phase of 
respiration during examination [16]. The inter-observer agreement on the EG Scan diagnosis of medium/large OV was moderate $(\mathrm{K}=0.47)$, while agreement on small OV was slight $(\mathrm{k}=0.19)$. Interestingly, these patterns were similar in case of $\mathrm{C}-\mathrm{OGD}$ ( $\mathrm{K}$ for medium/large $\mathrm{OV}=0.67$ and $\mathrm{K}$ for small $\mathrm{OV}=0.22$ ). These values suggest that the performance of both procedures in detecting small OV varies significantly across different observers. Moreover, they draw attention to the fact that C-OGD may not be an objective reference standard in the setting of small OV. These findings may need to be confirmed in a larger study which should also examine whether agreement varies between observers with variable levels of experience. We could not investigate this parameter due to the small number of videos included in our study. Our results are comparable to other studies reporting poor agreement on C-OGD findings of small OV compared to medium/large OV [17]. Nevertheless, data from C-OGD interobserver agreement studies should be interpreted with caution, because of the variation in classification systems, assessment methods (still images vs. video recordings) and number as well as expertise of observers [18]. Moreover, all C-OGD studies were performed in the 1980's and early 1990's using outdated imaging technologies with a significantly inferior image quality $[19,20]$.

The accuracy of conventional TNE (non-portable and non-disposable) in the detection of OV has been evaluated in a recent study by Choe et al, [7] where the authors reported excellent sensitivity and specificity of $100 \%(95 \% \mathrm{Cl}$ 93\%-100\%) and $93 \%(95 \% \mathrm{Cl} \quad 79 \%-98 \%)$, respectively, for the detection of any OV. All patients underwent TNE and C-OGD within two weeks by two different operators, however, the diagnosis of whether OV were present or absent was made on the basis of post-hoc review of still images from both procedures by three blinded endoscopists. This type of study design may lead to significant bias because both index test and reference standard results were interpreted by the same individuals $[9,21]$.

\section{Study strengths and limitations}


This pilot study had strengths and limitations. The trial design, conduct, and reporting conforms to current guidelines for undertaking diagnostic accuracy studies which is an important strength $[9,21]$. We selected a consecutive group of patients who are representative of those for which the test will be used in clinical practice. Although the EG Scan (index test) and C-OGD (reference standard) operators were blinded to the findings of either tests, it remains difficult to rule out operator bias since the investigators were not blinded to the intervention, nevertheless, this limitation is true of any endoscopic study. Moreover, all the EG Scan procedures were performed by a single endoscopist (SSS) with an in interest in the TNE technique. We attempted to address this by examining the interobserver agreement on the EG Scan diagnosis among other endoscopists with varying levels of expertise. Similarly, participation bias may also exist, since patients who agree to undergo TNE may be more motivated to tolerate the procedure and find it acceptable.

We used validated VAS [10] and 3-item (EG Scan or C-OGD or either) questionnaires to measure tolerability and preference, respectively. Day 0 questionnaires were administered by the trial coordinator face to face in order to improve response rate $(100 \%)$ and minimize response bias (0\% non-responders) [22], while day 14 were self-administered questionnaires to help minimize interviewer bias [22]. The response rate on day 14 was $87 \%$ (39 out of 45) for VAS (table 2) and 82\% (37 out of 45) for preference (figure 2), therefore response bias remains unlikely. Furthermore, in the latter questionnaire, patients were offered the choice of "either" for their procedure preference to minimize forced choice bias, where respondents who have no preference will be forced to select an answer (EG Scan or C-OGD) that may or may not reflect their true feelings [22]. Finally, we cannot rule out social desirability or obsequiousness bias where respondents may alter their questionnaire responses in the direction they perceive to be desired by the investigator [22], however, this limitation pertains to the majority of questionnaire study designs. Patients were offered neither monetary compensation nor any other incentive (such as an earlier appointment) to participate in the study. 


\section{Implications for clinical practice}

A major limitation of the currently available transnasal endoscopes is the lack of portability and the requirement for decontamination facilities. Hence, TNE procedures have, so far, been performed in the endoscopy unit and not routinely used for OV screening, possibly because endoscopists perceive little added value in performing TNE when C-OGD is readily available and can simultaneously be utilized for variceal band ligation if necessary [7]. Screening for OV using C-OGD is reportedly under-implemented in clinical practice, in particular, within the community hospitals setting [23]. Hepatologists were more likely to screen patients compared to general gastroenterologists and more likely to initiate primary prophylaxis [23].

The EG Scan may address these limitations by offering a portable and disposable endoscopic imaging tool that can be used as a pre-screening test in the office to reduce the number of unnecessary endoscopies and potentially improve screening uptake by offering a well tolerated and more preferred test to patients. Moreover, this strategy may facilitate better targeting of C-OGD resources for patients who are shown to have OV on EG scan and will therefore require interventional measures such as variceal band ligation or betablocker therapy. Data from our study will help inform future trials to evaluate the costeffectiveness of EG Scan screening followed by C-OGD only when OV are detected compared to the current strategy of universal C-OGD screening.

The EG Scan provides direct visualization of the oesophagus with a binary outcome similar to the interpretation of C-OGD, but it also has limitations compared to other non-invasive markers such as blood tests, in particular, the EG Scan is operator dependent and requires training and expertise for the diagnosis and grading of OV which determines the need for prophylaxis. These factors may limit its wide spread use especially by nongastroenterologists in the community. Nonetheless, office-based TNE can be performed successfully by physician extenders after a short training program and the learning curve for 
the detection of OV remains to be determined in future larger studies [24]. The risk of epistaxis will also need to be carefully weighed when using this technique in cirrhotic patients.

\section{Conclusions}

The EG Scan screening in this pilot study of patients with liver cirrhosis was feasible, safe, and remained highly acceptable compared to C-OGD 14 days after procedures. EG Scan accuracy was high for the detection of clinically significant medium/large OV, but this reduced when small OV were included. This is could be due to the poor interobserver agreement on the diagnosis of small OV using either EG Scan or C-OGD. As a result, utilizing accuracy alone as a study endpoint may not be adequate. Future studies should focus on the effectiveness of EG Scan in routine clinical practice and cost effectiveness in the overall health care system. Important effectiveness endpoints of a new OV screening strategy could be the number of C-OGDs saved; or the proportion of variceal bleeds or deaths prevented compared to the current standard of care [25].

\section{Acknowledgements}

We would like to acknowledge the facility, staff, and equipment support by the NIHR Nottingham Digestive Diseases Biomedical Research Unit, Nottingham, United Kingdom. 
Table 1: Baseline characteristics of the study cohort $(n=45)$. Data presented as number $(\%)$ or mean (+/- standard deviation (SD)) or median (interquartile range (IQR))

\begin{tabular}{|c|c|}
\hline Variable & Value \\
\hline Age & $59(+/-11)$ \\
\hline Male gender & $33(73.3 \%)$ \\
\hline White ethnicity & $45(100 \%)$ \\
\hline \multicolumn{2}{|l|}{ Aetiology of cirrhosis } \\
\hline Alcohol & $19(42.2 \%)$ \\
\hline Non-alcoholic fatty liver disease & $12(26.7 \%)$ \\
\hline Hepatitis C virus & $3(6.7 \%)$ \\
\hline Hepatitis C virus and alcohol & $2(4.4 \%)$ \\
\hline Hereditary Haemochromatosis & $4(8.9 \%)$ \\
\hline Other (AlH n=2, PBC n=2, Unknown $n=1)$ & $5(11.1 \%)$ \\
\hline \multicolumn{2}{|l|}{ Child Pugh class } \\
\hline $\mathrm{A} / \mathrm{B} / \mathrm{C}$ & $34(75.6 \%) / 10(22.2 \%) / 1(2.2 \%)$ \\
\hline Child Pugh score & $6.2(+/-1.3)$ \\
\hline \multicolumn{2}{|l|}{ Oesophageal varices } \\
\hline Yes & $22(49 \%)$ \\
\hline Small (grade1) & $10(46 \%)$ \\
\hline Medium/large (grade 2/3) & $12(54 \%)$ \\
\hline No & $23(51 \%)$ \\
\hline Gastric varices & $3(6.7 \%)$ \\
\hline Prothrombin time & $12.3(+/-1.9)$ \\
\hline Platelet count & 134 (IQR 89-196) \\
\hline Spleen diameter & $13.1(+/-2.1)$ \\
\hline Beta-blocker use & $10(22.2 \%)$ \\
\hline
\end{tabular}




\begin{tabular}{|l|l|}
\hline Co-morbidities & \\
\hline Diabetes & $15(33.3 \%)$ \\
\hline Hypertension & $15(33.3 \%)$ \\
\hline Chronic obstructive pulmonary disease & $7(15.6 \%)$ \\
\hline Ischemic heart disease & $4(8.9 \%)$ \\
\hline Screening indication & $27(60 \%)$ \\
\hline Surveillance indication & $18(40 \%)$ \\
\hline
\end{tabular}

$\mathrm{AIH}$, autoimmune hepatitis; $\mathrm{PBC}$, primary biliary cirrhosis 
Table 2: Quality, safety, and acceptability of endoscopic assessments. Data presented as number (\%) or mean (+/- standard deviation (SD)) or median (interquartile range (IQR)).

\begin{tabular}{|c|c|c|c|}
\hline Variable & EG Scan & C-OGD & P value \\
\hline Rate of successful intubation & $47(94 \%)$ & $48(96 \%) \S$ & 0.882 \\
\hline Rate of complete evaluation & $44(88 \%)$ & $46(92 \%) \dagger$ & 0.411 \\
\hline \multicolumn{4}{|l|}{$\begin{array}{l}\text { Adverse events scales } \\
(0=\text { none, } 10=\text { worst })\end{array}$} \\
\hline Gagging/retching & 1 (IQR 0-3) & 4 (IQR 0-8) & $<0.001$ \\
\hline Choking & 0 (IQR 0-2) & 0.5 (IQR 0-5 & $<0.001$ \\
\hline Discomfort & 2 (IQR 0-3) & 3 (IQR 0-7) & 0.011 \\
\hline Nasal pain (EG Scan only) & 0 (IQR 0-1) & - & \\
\hline Preference day 0 total $(n=34) \ddagger$ & $26(76.5 \%)$ & $8(23.5 \%)$ & $<0.001$ \\
\hline sedation subgroup $(n=20)$ & $13(65 \%)$ & $7(35 \%)$ & $<0.001$ \\
\hline no sedation subgroup $(\mathrm{n}=14)$ & $13(92.9 \%)$ & $1(7.1 \%)$ & $<0.001$ \\
\hline VAS score day 0 total $(n=45)$ & $7.8(+/-2.2)$ & $6.8(+/-3.0)$ & 0.058 \\
\hline sedation subgroup $(\mathrm{n}=26)$ & $7.4(+/-2.6)$ & $7.9(+/-2.3)$ & 0.477 \\
\hline no sedation subgroup $(\mathrm{n}=19)$ & $8.4(+/-1.4)$ & $5.2(+/-3.3)$ & $<0.001$ \\
\hline Preference day 14 total $(n=27) f$ & $21(77.8 \%)$ & $6(22.2 \%)$ & $<0.001$ \\
\hline sedation subgroup $(n=16)$ & $11(68.8 \%)$ & $5(31.2 \%)$ & $<0.001$ \\
\hline no sedation subgroup $(\mathrm{n}=11)$ & $10(90.9 \%)$ & $1(9.1 \%)$ & 0.001 \\
\hline VAS score day 14 total $(n=39) \uparrow$ & $7.0(+/-2.3)$ & $5.0(+/-3.2)$ & $<0.001$ \\
\hline sedation subgroup $(\mathrm{n}=22)$ & $6.4(+/-2.6)$ & $5.8(+/-3.1)$ & 0.383 \\
\hline no sedation subgroup $(\mathrm{n}=17)$ & $7.8(+/-1.5)$ & $4.0(+/-3.2)$ & $<0.001$ \\
\hline
\end{tabular}

C-OGD, conventional oesophagogastroduodenoscopy. § C-OGD failed in two patients (not tolerated on two attempts by one patient and the other had a very high systolic blood pressure of $230 \mathrm{mmHg}$ ). $\dagger$ Two patients withdrew consent halfway through the procedure. $\ddagger n=34 / 45$ expressed preference for either EG Scan or C-OGD while the remaining 11 expressed no preference (either). f $n=27 / 39$ expressed preference for either EG Scan or 
C-OGD while the remaining 12 expressed no preference (either). I $n=39 / 45$ (87\%) returned the 14-day questionnaire and reported VAS scores.

\section{Figure legends}

Figure 1: The EG Scan ${ }^{\mathrm{TM}}$ II system. A) the portable case with four main parts; B) the image processor (top left), disposable probe (top right), air tube (bottom right) and hand-held controller (bottom left); C) the system connected and ready for use; D) close view of the capsule probe tip.

Figure 2: Study flowchart. OV, oesophageal varices; C-OGD, conventional oesophagogastroduodenoscopy; SBP, systolic blood pressure. † 5 cases false positive on EG Scan all were graded as small OV; $\ddagger$ EG Scan missed 4 cases of OV (small $n=3$, medium/large $n=1$ ).

Figure 3: Procedure preference (patients were asked which procedure they would prefer to have in the future if clinically indicated). C-OGD, conventional oesophagogastroduodenoscopy.

Figure 4: Procedure experience measured at day 0 and day 14 using a 10-point visual analogue scale $(0=$ worst, $10=$ best $)$. Thick horizontal line represents median, hollow diamond symbol represents mean. C-OGD, conventional oesophagogastroduodenoscopy.

Figure 5: Example findings of EG Scan. A) Normal oesophagus; B) Grade 1/small oesophageal varices (OV); C) Grade 2/medium OV; D) Grade 3/large OV; E) OV with red spots; F) Gastric varix on retroflexion view. 


\section{References}

1. Garcia-Tsao G, Sanyal AJ, Grace ND, Carey W. Prevention and management of gastroesophageal varices and variceal hemorrhage in cirrhosis. Hepatology 2007; 46: 922-938

2. Tripathi D, Stanley AJ, Hayes PC, Patch D, Millson C, Mehrzad H et al. UK guidelines on the management of variceal haemorrhage in cirrhotic patients. Gut 2015 Apr 17 [Epub ahead of print], DOI:

3. Spiegel BM, Targownik L, Dulai GS, Karsan HA, Gralnek IM. Endoscopic screening for esophageal varices in cirrhosis: Is it ever cost effective? Hepatology 2003; 37: 366-377

4. Kovalak M, Lake J, Mattek N, Eisen G, Lieberman D, Zaman A. Endoscopic screening for varices in cirrhotic patients: data from a national endoscopic database. Gastrointest Endosc 2007; 65: 82-88

5. Rye K, Scott R, Mortimore G, Lawson A, Austin A, Freeman J. Towards noninvasive detection of oesophageal varices. Int J Hepatol 2012; 2012: 343591

6. Abraham NS, Fallone CA, Mayrand S, Huang J, Wieczorek P, Barkun AN. Sedation versus no sedation in the performance of diagnostic upper gastrointestinal endoscopy: a Canadian randomized controlled cost-outcome study. Am J Gastroenterol 2004; 99: 1692-1699

7. Choe WH, Kim JH, Ko SY, Kwon SY, Kim BK, Rhee KH et al. Comparison of transnasal small-caliber vs. peroral conventional esophagogastroduodenoscopy for evaluating varices in unsedated cirrhotic patients. Endoscopy 2011; 43: 649-656

8. Aedo MR, Zavala-Gonzalez MA, Meixueiro-Daza A, Remes-Troche JM. Accuracy of transnasal endoscopy with a disposable esophagoscope compared to conventional endoscopy. World J Gastrointest Endosc 2014; 6: 128-136

9. Bossuyt PM, Reitsma JB, Bruns DE, Gatsonis CA, Glasziou PP, Irwig LM et al. Towards complete and accurate reporting of studies of diagnostic accuracy: The STARD Initiative. Ann Intern Med 2003; 138: 40-44 
10. Likert R. A technique for the measurement of attitudes. Archives of Psychology 1932; 22: $1-55$

11. Fleiss JL. Measuring nominal scale agreement among many raters. Psychological Bulletin 1971; 76: 378-382

12. Landis JR, Koch GG. The measurement of observer agreement for categorical data. Biometrics 1977; 33: 159-174

13. Sami SS, Subramanian V, Fernández-Sordo JO, Saeed A, Singh S, lyer PG et al. Performance characteristics of unsedated ultrathin video endoscopy in the assessment of the upper gastrointestinal tract: systematic review and meta-analysis. Gastrointest Endosc 2015 Sep 11 [Epub ahead of print], DOI: 10.1016/j.gie.2014.05.114:

14. Guturu P, Sagi SV, Ahn D, Jaganmohan S, Kuo YF, Sood GK. Capsule endoscopy with PILLCAM ESO for detecting esophageal varices: a meta-analysis. Minerva Gastroenterol Dietol 2011; 57: 1-11

15. White CM, Kilgore ML. PillCam ESO versus esophagogastroduodenoscopy in esophageal variceal screening: A decision analysis. J Clin Gastroenterol 2009; 43: 975981

16. Sarin SK, Lamba GS, Kumar M, Misra A, Murthy NS. Comparison of endoscopic ligation and propranolol for the primary prevention of variceal bleeding. The New England journal of medicine 1999; 340: 988-993

17. Calès P, Oberti F, Bernard-Chabert B, Payen J-L. Evaluation of Baveno recommendations for grading esophageal varices. Journal of Hepatology 2003; 39: 658659

18. Winkfield B, Aube C, Burtin P, Cales P. Inter-observer and intra-observer variability in hepatology. Eur J Gastroenterol Hepatol 2003; 15: 959-966

19. Bendtsen F, Skovgaard LT, Sorensen TI, Matzen P. Agreement among multiple observers on endoscopic diagnosis of esophageal varices before bleeding. Hepatology 1990; 11: 341-347 
20. [Anonymous]. North Italian Endoscopy Club for the Study and Treatment of Esophageal Varices. Prediction of the first variceal hemorrhage in patients with cirrhosis of the liver and esophageal varices. A prospective multicenter study. The New England journal of medicine 1988; 319: 983-989

21. Whiting PF, Rutjes AW, Westwood ME, Mallett S, Deeks JJ, Reitsma JB et al. QUADAS2: a revised tool for the quality assessment of diagnostic accuracy studies. Ann Intern Med 2011; 155: 529-536

22. Choi BC, Pak AW. A catalog of biases in questionnaires. Prev Chronic Dis 2005. [accessed: 2nd December 2015]. Available from: http://www.cdc.gov/pcd/issues/2005/jan/04 0050.htm.

23. Barritt ASt, Arguedas MR. Practice patterns in screening for varices: an American survey. Dig Liver Dis 2009; 41: 676-682

24. Alashkar B, Faulx AL, Hepner A, Pulice R, Vemana S, Greer KB et al. Development of a Program to train Physician Extenders to Perform Transnasal Esophagoscopy and Screen for Barrett's Esophagus'. Clin Gastroenterol Hepatol 2014; 12: 785-792

25. Ferrante di Ruffano L, Hyde CJ, McCaffery KJ, Bossuyt PM, Deeks JJ. Assessing the value of diagnostic tests: a framework for designing and evaluating trials. BMJ 2012; 344: e686 